\title{
In-Vivo IVUS Tissue Classification: A Comparison Between RF Signal Analysis and Reconstructed Images
}

\author{
Karla L. Caballero ${ }^{1}$, Joel Barajas ${ }^{1}$, Oriol Pujol ${ }^{1,2}$, \\ Neus Salvatella ${ }^{3}$, and Petia Radeva ${ }^{1}$ \\ ${ }^{1}$ Computer Vision Center, UAB Bellaterra Barcelona 08193, Spain, \\ ${ }^{2}$ Dept. Matemàtica Aplicada i Anàlisi. University of Barcelona, Barcelona, Spain \\ ${ }^{3}$ Hospital Universitari German Trias i Pujol, Badalona, Spain \\ klcaballero@cvc.uab.es
}

\begin{abstract}
In this paper we present a novel framework for classification of the different kind of tissues in intravascular ultrasound (IVUS) data. We expose a normalized reconstruction of the IVUS images from radio frequency (RF) signals, and the use of these signals for classification. The reconstructed data is described in terms of texture based features and feeds an ECOC-Adaboost learning process. In the same manner, the RF signals are characterize using Autoregressive models, and classified with a similar learning process. A comparison is performed among these techniques and with DICOM based classification ones obtaining very promising results.
\end{abstract}

\section{Introduction}

Plaque rupture is one of the most frequent cause of acute coronary syndromes. Most of them end in myocardial infarction or sudden cardiac death. Many studies, nowadays, report a high correlation between multiple plaque ruptures and acute coronary syndrome. To understand the mechanisms of plaque destabilization and guide a pharmacological treatment, it is of high interest the characterization of the fragile part of the atheromatous plaque and to differentiate between low-risk and high-risk plaques.

Intravascular ultrasound (IVUS) offers a unique view of the morphology of the arterial plaque, it displays the morphology and histological properties of a cross-section of the vessel. It is generally accepted that in these images three are the different kind of plaque tissues distinguishable: calcium formations (characterized by a very high echo-reflectivity and absorbtion); fibrous plaque (medium echo-reflectivity and good transmission coefficient) and lipidic or soft plaque (very low reflectance). The automatic analysis of the IVUS images is of vital importance to the coronary community, since it allows feasible ways to predict vulnerable plaques as well as to quantify the amount of the different tissues, avoiding all the subjectivity due to the physician who performs the study and the amount of hours needed to label the recorded IVUS pullbacks. 
The analysis of IVUS data is approached in two ways in literature: On one hand, several authors propose the analysis of the DICOM images themselves by means of normalization procedures and texture analysis 1513. On the other hand, several researchers prefer the use of the original radio-frequency signal when available 54 . However, until now there is no evidence of one of the techniques being better than the other.

In this paper we propose two methods that exploit the advantages of both trends of work: we propose a framework to reconstruct normalized IVUS images from the radio frequency signal and to apply the image based characterization process. This data is feeded into an ECOC improved Adaboost framework to perform the final classification. The main advantages of our method are threefold: firstly, our method offers the advantage of normalizing all cases to a fixed parameter set. Secondly, it allows to use very complex texture-based descriptors. And thirdly, by using a machine learning technique we ensure a proper behavior of the classification approach. In addition, this article presents an approach of tissue classification using RF signals with a similar learning machine technique. Then a comparison between the classification using reconstructed normalized IVUS images with different settings and RF signal analysis is performed.

In section 2 , we present an image reconstruction process, in section 3 , it is explained an RF signal analysis and characterization. In section 4, it is exposed how to extract the different texture features used in our study. Finally, the results, discussion, and conclusion are presented.

\section{Image Reconstruction Process}

An IVUS equipment consists of a main computer to reconstruct images, and a catheter which is introduced into the vessel to perform an exploration. This catheter carries an ultrasound emitter which shots a given number of beams, and a transducer that collects their reflections as RF signals. Based on the kind of tissue, these signals vary their frequency and amplitude. There is enough evidence that these signals are suitable to perform a classification process [9:5].

\subsection{RF Signal Acquisition}

The RF signals are acquired using a 12-bit Acquiris acquisition card with a sampling rate of $200 \mathrm{MHz}$. The frequency of the catheter transducer is $40 \mathrm{Mhz}$ for our data, and it is assumed a sound speed in tissue of $1565 \mathrm{~m} / \mathrm{s}$. Each IVUS image consists of a total of 256 A-lines (ultrasound beams), and a length of $6.5 \mathrm{~mm}$. Thus, RF data were acquired from patient pullback sequences in vivo.

\subsection{Image Construction}

Having the acquired RF data, an image construction framework is applied to obtain the IVUS images with the same parameters set. Hence as a preprocessing step, the acquired signals are filtered with a band pass filter with $50 \%$ of gain 
located at the transducer frequency. Additionally, a time gain compensation is applied in order to correct the tissue attenuation $\alpha=1 \mathrm{DbMhz} / \mathrm{s}$.

After the signals are compensated a signal envelope is calculated using the Hilbert transform. This envelope is normalized in a range from 0 to 1 , and compressed in a logarithmical form. This is done in order to distribute the gray levels in the histogram and also to enhance the image visualization.

The image is constructed in cartesian form and the missing pixels between each angle are filled using bilinear interpolation. Then, a non linear Digital Development Process (DDP) to regulate the contrast radially is applied. The parameters are fixed in order to normalize all the images with the same contrast. This process allow us to change the contrast of the image easily with low computational cost, which is not an easy task in the DICOM images since the construction parameters are not usually available. Figure 1 shows an example of constructed images with different gains.

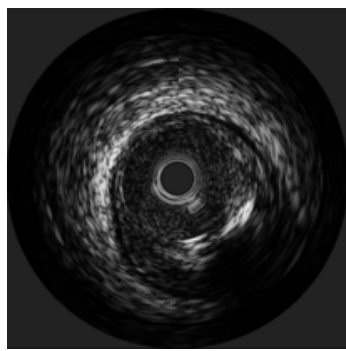

(a)

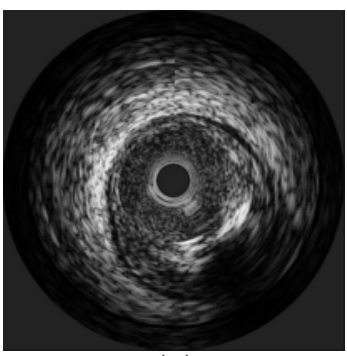

(b)

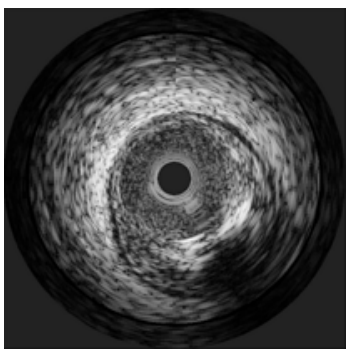

(c)

Fig. 1. Reconstructed IVUS images from RF signals with different DDP gain parameters. (a)DDP gain parameter fixed to 1.04. (b)DDP gain parameter fixed to 2.20 . (c)DDP gain parameter fixed to 3.20 .

The constructed images are used mainly for: classification with a texture based approach, visualization as a reference for the RF signal correlation, and for manual segmentation used in the training set.

\section{RF Signal Analysis}

Once the RF signals have been acquired, filtered, and compensated, a characterization process is performed. According to [8], one of the most suitable methods to analyze ultrasound signals is the use of Autoregressive (AR) models of their power spectrum. Thus, in this approach the utilization of AR models is explored.

\subsection{Autoregressive Models}

The autoregressive models can be defined as a linear prediction formula where the output $x$ at a certain point $n$ is equal to a linear combination of its previous outputs $p$ with a given weight $a_{p}[12$. 


$$
x(n)=\sum_{k=1}^{p} a_{p}(k) x(n-k),
$$

The AR models are used to approximate the power spectrum of a RF signal window. In our case it is composed of 64 samples, and 12 of the $256 \mathrm{~A}$-lines. The Burg method is used to estimate the power spectrum, and the optimal model order (21) was obtained using the Akaike's Final Error Prediction 6]. Then, only one side of the spectrum is used because of its symmetry. It is composed of 200 sampled frequencies ranging from 0 to $100 \mathrm{MHz}$. Thus, each frequency value of the model is used as a signal feature for classification, as it is shown in figure 2.

Here an sliding window of 64 samples and 12 A-lines, and a displacement of 16 samples and 4 A-lines are used for characterization. These is done in order to increase the resolution of the characterization. Note that this window corresponds to a region of $16 \times 12$ pixels in the reconstructed polar image.

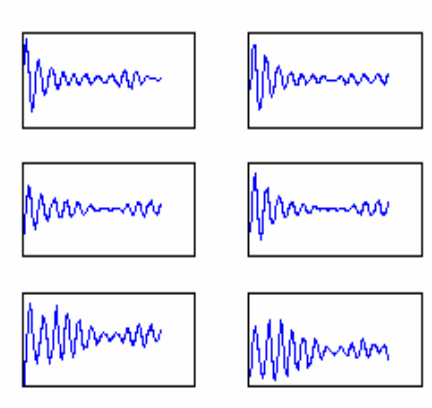

(a)

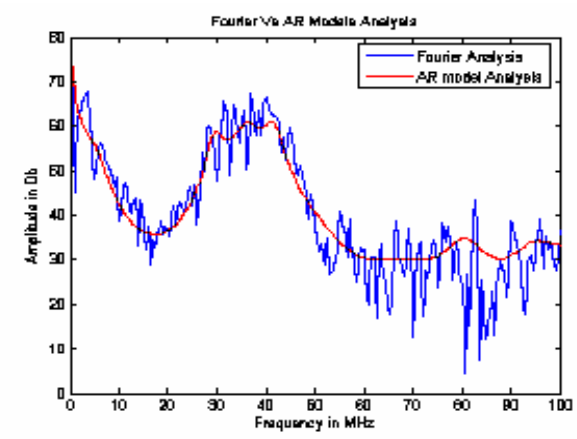

(b)

Fig. 2. The use of a different RF signal sections (a) to create an Autoregressive $\operatorname{model}(\mathrm{b})$

\section{Texture Features Extraction}

\subsection{Co-occurrence Matrix}

The co-occurrence matrix can be defined as an estimation of the joint probality density function of gray level pairs in a image 11. The element values in a matrix are bounded from 0 to 1 and the sum of all element values is:

$$
P(i, j, D, \theta)=P(I(l, m)=i \otimes I(l+D \cos (\theta), m+D \sin (\theta))=j),
$$

where $I(l, m)$ is the gray value at the $\operatorname{pixel}(l, m), D$ is the distance among pixels and $\theta$ is the angle of each of neighbors. The angle orientation $\theta$ has been fixed to be $\left[0^{\circ}, 45^{\circ}, 90^{\circ}, 135^{\circ}\right]$, because, according to 311 , it is the minimum set of orientations needed to describe a second-order statistic measures of texture. After computing this matrix some characterizing measures are extracted such as energy, entropy, the Inverse Difference Moment, shade, inertia and Promenance, 
as are defined in 11. Thus, a 48 feature space is built to perform the classification process for each pixel, since we are estimating 6 different measures at 4 orientations and two distances $D=[5,8]$.

\subsection{Local Binary Patterns}

These feature extractor operators are used to detect uniform texture patterns into circular neighborhoods with any quantization of angular space and spatial resolution 10 . It is based on a circular symmetric neighborhood of $P$ members of a circle with radius $R$. In order to achieve gray level invariance, the central pixel $g_{c}$ is subtracted to each neighbor $g_{p}$, assigning to the result 1 if the difference is positive and 0 if it is negative. Each neighbor is weighted with a $2^{p}$ value. Then, the neighbors are added, and the result is assigned to the central pixel.

$$
L B P_{R, P}=\sum_{p=0}^{P} s\left(g_{p}-g_{c}\right) \cdot 2^{p}
$$

The application of this operator generates a 3 dimensional space in texture analysis, by applying a radius of $R=[1,2,3]$ and a neighborhood of $P=[8,16,24]$.

\subsection{Gabor Filters Bank in Texture Analysis}

The Gabor Filters is an special case of wavelets [12], and is essentially a Gaussian $g$ modulated by a complex sinusoid $s$. In 2D, a Gabor filter has the following form in the spatial domain:

$$
h(x, y)=\frac{1}{2 \pi \sigma_{x^{\prime}} \sigma_{y^{\prime}}} \exp \left\{-\frac{1}{2}\left[\left(\frac{x^{\prime}}{\sigma_{x^{\prime}}}\right)^{2}+\left(\frac{y^{\prime}}{\sigma_{y^{\prime}}}\right)^{2}\right]\right\} \cdot s(x, y),
$$

where $s(x, y)$ and the Gaussian rotation are defined as:

$$
\begin{aligned}
& s(x, y)=\exp [-i 2 \pi(U x+V y)] \\
& x^{\prime}=x \cos \theta+y \sin \theta, y^{\prime}=-x \sin \theta+y \cos \theta .
\end{aligned}
$$

$x^{\prime}$ and $y^{\prime}$ represent the spatial coordinates rotated by an angle $\theta . \sigma_{x^{\prime}}$ and $\sigma_{y^{\prime}}$ are the standard deviations for the Gaussian envelope. An aspect ratio $\lambda$ and its orientation are defined as:

$$
\lambda=\frac{\sigma_{x^{\prime}}}{\sigma_{y^{\prime}}}, \phi=\arctan V / U
$$

where $U$ and $V$ represent the 2D frequencies of the complex sinusoid.

We have fixed $\lambda=1$ in order to manage with isotropic gaussian envelopes, provoking that both $\sigma_{x^{\prime}}$ and $\sigma_{y^{\prime}}$ are equal, leading to discard $\theta$. Then, $\theta=0$. We have represented the $2 \mathrm{D}$ frequency, $(U, V)$, by a polar representation $F, \phi$. Thus, we have created a filter bank using the following parameters:

$$
\begin{aligned}
& \sigma_{x}=\sigma_{y}=[12.7205,6.3602,3.1801,1.5901] \\
& \quad \phi=\left[0^{\circ}, 45^{\circ}, 90^{\circ}, 135^{\circ}\right], \\
& F=[0.0442,0.0884,0.1768,0.3536] \\
& \text { yielding a } 16 \text { dimensional space for each pixel. }
\end{aligned}
$$




\subsection{Feature Vector Generation}

Now, we have extracted all the features explained above. We have compiled them into a unique feature vector of 67 dimensions for each pixel, which will be used to train the classifier. The main idea is to extract the best of each technique in order to improve the classification performance.

\section{Classification}

Once we have designed a characterization framework for images using texture and for RF signals using AR models, a classification scheme is developed. We have established 3 classes of tissue: fibrotic plaque, lipid or soft plaque, and calcium. Since we already know how many classes are, and how they behave, we use the Adaptive Boosting (Adaboost) supervised learning technique.

Adaboost is a method that allows us to add "weak" classifiers until some desired training error is obtained [1716]. In each step of the algorithm a weight is assigned to each feature, which means how accurate this feature can classify the training data. It results into a linear combination of weaker classifiers, and the weight of each one depends on the amount of data it can classify.

Since we have a multiclass problem, we need to establish some combination criteria for the classifier output. Firstly, we used decision tables to combine the outputs but the appearance of draws was common. As a result, we switched to use Error Correction Output Codes (ECOC) 14. Basically, it consists in assigning a code map table which relates classifiers outputs and classes. Then, the final classification is obtained finding the minimum distance between the resulting code and the classes.

The classification map from the ECOC is the same for both techniques. It is shown in table 1. Here, the number 0's indicate that these classes are not used in the selected classifier. Because there are only two classes left for each classifier, we apply one class versus the other, and not one versus the rest. The 1 's indicate that the classifier should output a positive value when this class is found, and negative one $(-1)$ when it is not.

Once we have all the classifiers results, it is found the Euclidean distance between each sample of the test and all the class codes. Thus, the class the minimum distance respect to the sample is assigned.

Table 1. ECOC code map used in the classification

\begin{tabular}{|c|c|c|c|}
\hline Classes & Classifier & 1 Classifier & 2 Classifier 3 \\
\hline Calcium & 1 & 1 & 0 \\
\hline Fibrotic Plaque & -1 & 0 & 1 \\
\hline Soft Plaque & 0 & -1 & -1 \\
\hline
\end{tabular}




\section{Results}

We have the RF signals from in vivo sequences, and their IVUS reconstructed images from a set of 10 patients all with the three classes of plaque. Each patient may have 1 or 2 vessel studies or pullbacks. Then, for each one, 10 to 15 different vessel sections are selected to be analyzed.

In order to avoid any kind of bias, the experiment has been repeated ten times by picking one different patient for testing and the rest for training in each iteration. This gives us a roughly idea of how the classification could work with a new unseen patient.

\subsection{Tissue Segmentation}

We have developed an application to construct IVUS images from the RF signals. It has the advantage of allowing the physicians to move the gain parameter of the DDP to simplify the manual segmentation task. Additionally, it permits the offline manipulation of the images for the physicians. Although the main propose of this step is to segment the training data and label it, the parameters used for the segmentation are stored for future analysis yielding to settings normalization.

The physicians have segmented from the vessel images around of 50 sections of interests per patient. These segmentations were taken as regions of interest (ROI) which were collected into a database categorized by patient. These were mapped in both RF signals and reconstructed images in order to build the data set for this experiment.

\subsection{RF Signal Classification}

One of the classifications we have done is the one based on the RF signals obtained from the ultrasound transducer. Here, we employ a classifier using the characterization of the signals based on AR models, as it is explained above. Then, we use the ROI selected by the physicians in order to train and test our system. The gentle Boost classifier with 50 stumps is utilized, because in our scheme all the frequencies have the same weight. Hence, the test set is classified, the classification error is calculated based on the number of misclassified samples of each class.

The average accuracy using AR models is $70 \%$ for the fibrous plaque, $77.2 \%$ for the soft and $75 \%$ for the calcium correctly classified. Although the accuracy is lower than some of the rates previously published using RF signals 877 , it is important to note that this experiment has been performed with a cross validation method. In addition, the test data is taken from different patients of the training data avoiding any kind of bias as in real performance. Notice that the accuracy using the training data is not shown since we think it is useless to measure the real performance of the system. 


\subsection{Texture Based Classification}

In order to test the performance of our texture based classification approach, we have selected 6 different DDP gain parameters to reconstruct the images. Then, there has been created five different data sets, and their extracted texture features have been processed separately using the characterization exposed before. For every DDP gain parameter values a classification error has been computed for each kind of tissue. Thus, the accuracy for different DDP gain parameters is shown in figure 3 ,

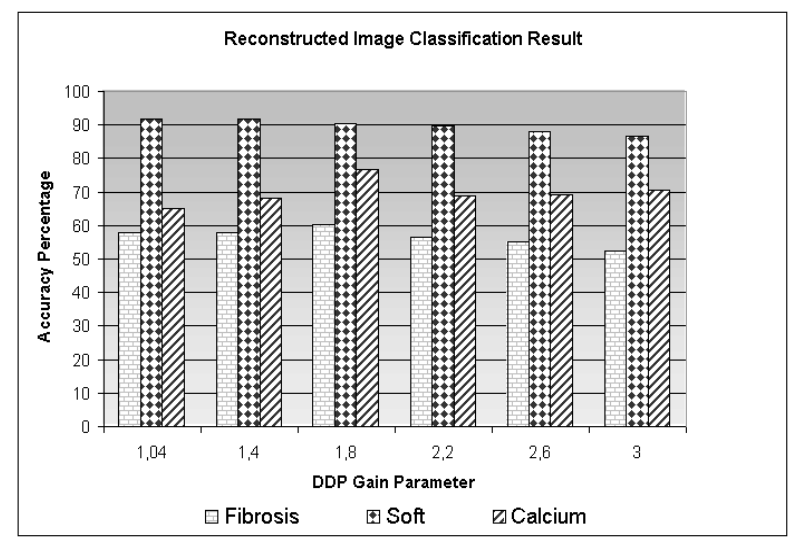

Fig. 3. Classification result among different DDP gain parameters for each type of tissue

It can be seen that the best gain parameter is 1.8 where the classification results are: $70 \%$ for fibrotic plaque, $90.5 \%$ for lipid, and $77.6 \%$ for calcium. Even when it can be chosen a certain gain as the best one for classification, a combination of multiple gain values, one designed for each tissue, could be suitable to improve the performance.

\subsection{Classification Comparison}

Having tested our classification using the two exposed characterization schemes, we show a comparative table of their performance in table 2. Notice that these results have been obtained with pixel resolution in both schemes, without any kind of postprocessing.

Additionally, a sample image classification result of both techniques is shown in figure 4 Here, a qualitative comparison can be observed. It is worth to mention that RF signal classification presents a border error. It could be caused by the physicians segmentation since it is easy to take little areas out of the real class. Because the RF characterization is based on the power spectrum, it can be easily influenced by the previous and after samples. Thus, this problem affects more in the RF signal based classification performance than in the texture based one. 
Table 2. Classification Results from RF Signal Analysis and Texture Based Analysis

\begin{tabular}{lcc}
\hline Classes & RF signal Analysis Texture & Base Analysis \\
\hline Fibrosis & $70 \%$ & $70 \%$ \\
Soft & $77 \%$ & $91.2 \%$ \\
Calcium & $75 \%$ & $77.6 \%$ \\
\hline
\end{tabular}

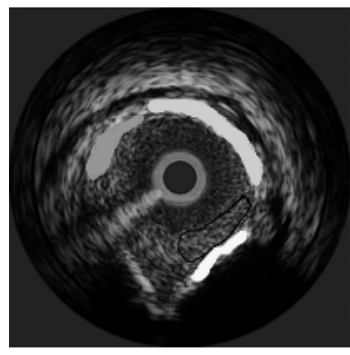

a

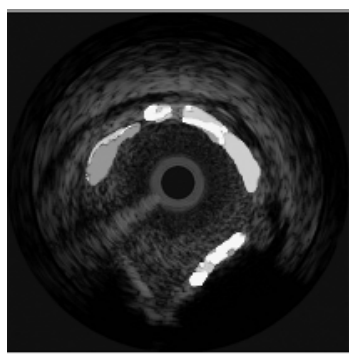

$\mathrm{b}$

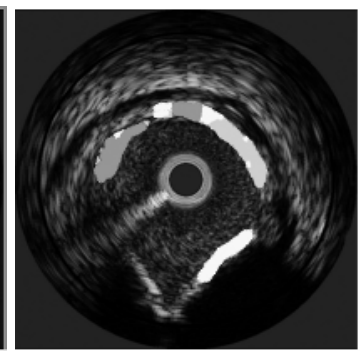

C

Fig. 4. Images (a) Segmented by the physician, white is calcium, light gray fibrosis and dark gray soft plaque. (b) Classified with RF signal Analysis. (c)Classified with Texture Based Features.

In any case, the accuracy rates shown here represents an improvement in the tissue characterization problem respect to the DICOM based approaches. Usually, the classifications rates reported in these approaches are around of $76 \%$ of the total performance without any kind of postprocessing 13 . The difference is mainly caused by the we use normalized data to test our classification framework.

\section{Discussion and Conclusion}

Two methods for tissue classification in IVUS from in vivo patients have been presented taking into account three types of plaque: fibrotic, soft, and calcium. We have proposed a more accurate technique based on a set of normalized data, which is not possible in conventional approaches based on DICOM images. To do so, an image construction method has been depicted.

The hit rate of the classification is higher in constructed images than in RF signal analysis. However, we suggest a combination of both characterization frameworks, because they provide complementary information. Thus, we are working now in the improvement of RF signal characterization since these represent the raw data and the real plaque response. Additionally, we have shown an application of a multi-class problem using ECOC to combine a set of two-class classifiers based on Gentle AdaBoost.

The classification exposed here has been done for each pixel but without any kind of postprocessing, since we desire to preserve the highest possible resolution. In order to improve the classification, some grouping techniques could be applied to the obtained results. 


\section{References}

1. J.G. Daugman. Uncertainty Relation for Resolution in Space, Spatial Frequency, and Orientation Optimized by Two-Dimensional Visual Cortical Filters. Journal of the Optical Society of America, 2(A):1160-1169, 1985.

2. Hans G. Feichtinger and Thomas Strohmer, editors. Gabor Analysis and Algorithms: Theory and Applications. Birkhäuser, 1998.

3. T. Randen J. H. Husoy. Filtering for texture classification: A comparative study. IEEE Transactions on Pattern Analysis and Machine Intelligence, 4:291-310, 1999.

4. T. Iwamoto, A. Tanaka, Y. Saijo, and M. Yoshizawa. Coronary plaque classification through intravascular ultrasound radiofrequency data analysis using self-organizing map. In IEEE Ultrasonics Symposium, pages 2054-2057, 2005.

5. A. Jeremias, M. Kolz, T. Ikonen, J. Gummert, and A. Oshima. Feasibility of in vivo intravascular ultrasound tissue characterization in the detection of early vascular transplant rejection. Circulation, 100:2127-2130, 1999.

6. S. Kay. Modern Spectral Estimation. Theory and Application. Prentice Hall, 1988.

7. A. Nair, D. Calvetti, and G. Vince. Regularized autoregressive analysis of intravascular ultrasound backscatter: Improvement in spatial accuracy of tissue maps. ieee transactions on ultrasonics, ferroelectrics, and frequency control, 51:420-431, 2004.

8. A. Nair, B. Kuban, E. Murat, P. Schoenhagen, S. Nissen, and D. Vince. Coronary plaque classification with intravascular ultrasound radiofrequency data analysis. Ciculation, 106:2200-2206, 2002.

9. A. Nair, B. D. Kuban, N. Obuchowski, and G. Vince. Assesing spectral algorithms to predict atherosclerotic plaque composition with normalized and raw intravascular ultrasound data. Ultrasound in Medicine $\&$ Biology, 27:1319-1331, 2001.

10. T. Ojala and M. Pietikainen T. Maenpaa. Multiresolution gray-scale and rotation invariant texture classification with local binary patterns. IEEE Transactions on Pattern Analysis and Machine Intelligence, 24:971-987, 2002.

11. R. Dubes P. Ohanian. Performance evaluation for four classes of textural features. Pattern Recognition, 25:819-833, 1992.

12. J. Proakis, C. Rader, F. Ling, and C. Nikias. Advanced Digital Signal Processing. Mc Millan, 1992.

13. O. Pujol. A semi-supervised Statistical Framework and Generative Snakes for IVUS Analysis. Graficas Rey, 2004.

14. O. Pujol, P. Radeva, and J. Vitria. Discriminant ecoc: A heuristic method for application dependent design of error correcting output codes. IEEE Transactions on Pattern Analysis and Machine Intelligence, 28:1001-1007, 2006.

15. O. Pujol, M. Rosales, and P. Radeva. Intravascular ultrasound images vessel characterization using adaboost. In Functional Imaging and Modelling of the Heart: Lecture Notes in Computer Science, pages 242-251, 2003.

16. R. Schapire. The boosting approach to machine learning: An overview, 2001.

17. Paul Viola and Michael Jones. Robust real-time object detection. In CVPR01, volume 1, page 511, 2001. 